\title{
Recognized Face Tracking for CONBE Robot
}

\author{
Sakmongkon Chumkamon \\ Department of Mechanical Information Science and Technology, Kyushu Institute of Technology, 680-4 Kawazu, \\ Iizuka, Fukuoka, Japan \\ Eiji Hayashi \\ Department of Mechanical Information Science and Technology \\ Kyushu Institute of Technology \\ 680-4 Kawazu, Iizuka-shi, Fukuoka, 820-8502, Japan \\ E-mail: haya@mse.kyutech.ac.jp \\ www.kyutech.ac.jp
}

\begin{abstract}
In our research, we develop the robot to combine with the consciousness and behavior which is the conscious and behavior robot (CONBE). In this paper we present the basis system in the CONBE robot that consist of the two main sections as the face recognition and robot gaze tracking. This system uses the face recognition using Fisherfaces that also refer to linear discriminant analysis (LDA). For the robot gaze tracking, we implement with the head of the CONBE robot that has a camera, two displays used for robot eyes and the actuators of 2 degrees of freedom. The actuators use to control the robot gaze for tracking the human face which the robot can recognize. In this paper, we experiment and present the results of the face recognition system and the tracking control system such as the tracking error and time.
\end{abstract}

Keywords: Consciousness-Based Architecture, Fisherfaces, Face recognition, Cognitive robot, face tracking.

\section{Introduction}

An autonomous robot requires efficient operation of their action. Decades, many good approaches have been proposed for controlling the autonomous robot under their condition such the development of the self-aware robot. Due to a robot with self-awareness has a realistic action to operate under the diversity situation more functional than a system without self-awareness. In this paper, we develop a conscious behavior robot (CONBEI) that imitates a human head. The mechanism of a head CONBE robot has 2 degrees of freedom that are orthogonal to each other in Cartesian coordinate. In our laboratory, we develop the robot to have the consciousness and behavior as the mention and propose the consciousness-based Architecture (CBA) system as a hierarchical structure model base on the mechanistic expressed model of animal consciousness and behavior [1]. The motivational action of animal is derived from recognition and comprehension respectively. Therefore, in this research, we focus on developing the recognition of the robot to be a basis of CBA system. We develop the face recognition with the CONBE robot to tracking face which is recognized. In face recognition, we use Fisher-faces algorithm that also refers to Linear Discriminant Analysis (LDA), use a video camera as an input instant image [2]. For hardware of the head robot, we use Dynamixel actuators that are controlled via RS485 bus to control the rotation of yaw and pitch axis of the head. For controlling, in the instant image, we determine the distances between the center of the tracking object and the center of camera image in vertical and horizontal axis. We then calculate the distances of the angular errors for each rotation in yaw 
axis and pitch axis and command the target angle to the actuators to tracking object. As previously mentioned, this research studies the face recognition that is one of recognition models in our CBA, and studies the part of the robot behavior that is an action of following gaze. This system imitates the animal character in the case of the animal seeing some people. It could recognize the persons and interested in them, it then acts some behavior such as a gaze following in our case. Our system imitating the animal system, due to our attempt is to increase the function of the robot.

The robot would operate more than following the command from human such as when the robot combines CBA system; it can introspect to act some suitable behavior when the robot can recognize the user being sad, happy and so forth. Moreover, this robot would be more attractive for human or user with this proposed.

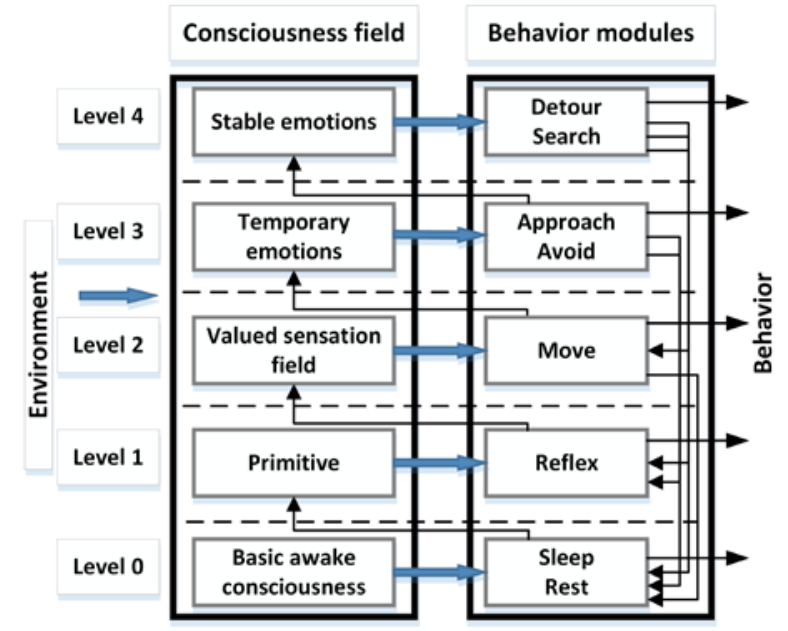

Fig.1. Consciousness-based architecture

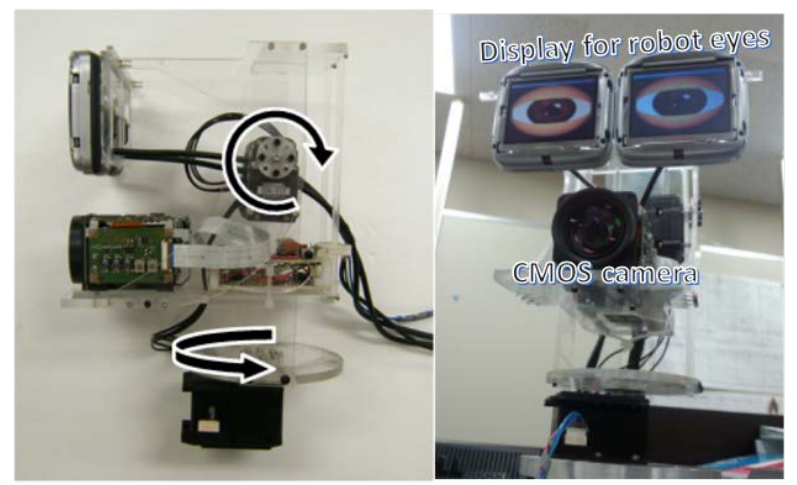

Fig.2. Head robot.

\section{Robot system overview}

The system based on CBA system combining with robot mechanism and software that we develop to archive face recognition and tracking face which is recognized. This system consists of two main such as hardware and software system.

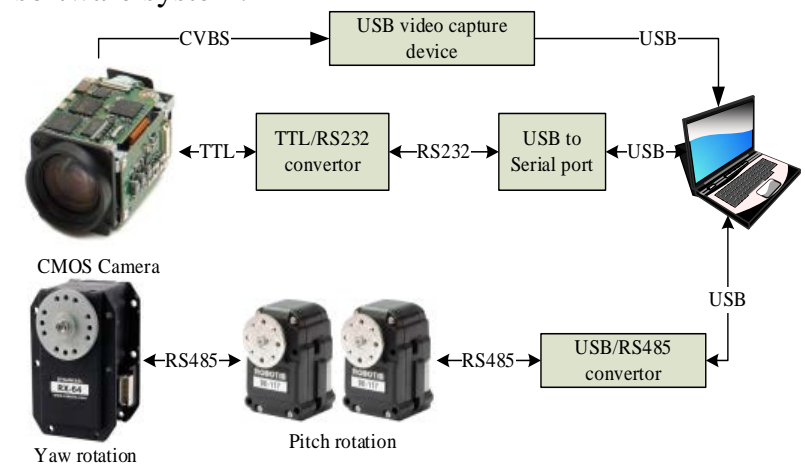

Fig.3. Connection diagram of the system.

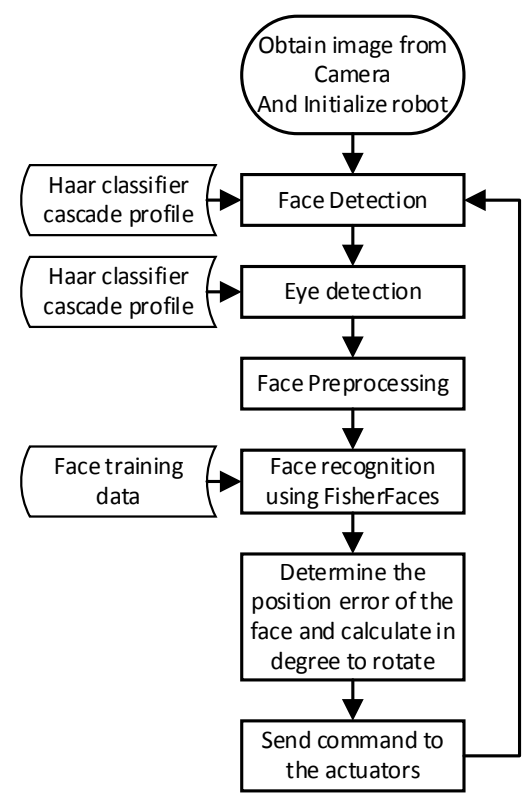

Fig.4. Software flow diagram.

\subsection{Hardware}

For head robot, we designed and constructed the structure with the acrylic plastic. The head robot also assembles with two actuators to rotate in Cartesian coordinate which the first actuator operates as a robot neck which base Dynamixel RX-64 and the second actuator for up down rotating which base Dynamixel DX-117. These actuators are DC gear motor with plastic 
body which are controlled by packet command containing with an identity number of the actuator via serial RS485 multi drop and yield the feedback such as position of rotation degree, temperature, load, Input voltage, speed and so forth. For obtaining the image to process, we use embedded CMOS camera based on SONY FCB-H11 which support full HD (1080i high definition) video format with; NTSL and PAL. The camera model is equipped with $\times 10$ optical auto focus zoom lens and the $\times 12$ digital zoom. The camera module can control the features of the camera with VISCA protocol via serial port. In this paper, we use this camera in PAL mode connecting with composite video channel and convert composite video channel to USB by USB video capture device. All the device in the robot system is controlled by a laptop which base CPU i7-2630QM, RAM 4GB.

\subsection{Software}

The system software has two major such face recognition and control which are implemented by $\mathrm{C}++$ language in a laptop. For the image processing library, this software use OpenCV library. At the beginning software from the face recognition, the system would capture the instant image from the camera then operating with human face detection using Haar features and Haar classifier cascade profile to determine the face position [3]. The software also detects the human eye due to obtain the definite position to provide the face preprocessing image due to the face recognition is vulnerable with some difference in lighting condition, face orientation, face expression, and so forth. For eye detection, it is used to crop the input image to show only the approximate eye region. We then perform the preprocessing image combining geometrical transformation and crop; separate histogram equalization for left and right side; and smoothing image using a bilateral filter; and elliptical mask to remain hair and background from the face image. Eventually we perform the face recognition using Fisherfaces algorithm with our training data and face label. Fisherfaces is the popular algorithm for face recognition using LDA which is to improve the capability of classification from PCA which is the core of the Eigenfaces method that is a powerful method to represent data. The simple concept of LDA is that the same classes should group closely together whereas different classes are more distance [4]. In this section we provide the environment for recognized face tracking. For the next section, we would experiment this system to apprehend the performance and other specific of this system.

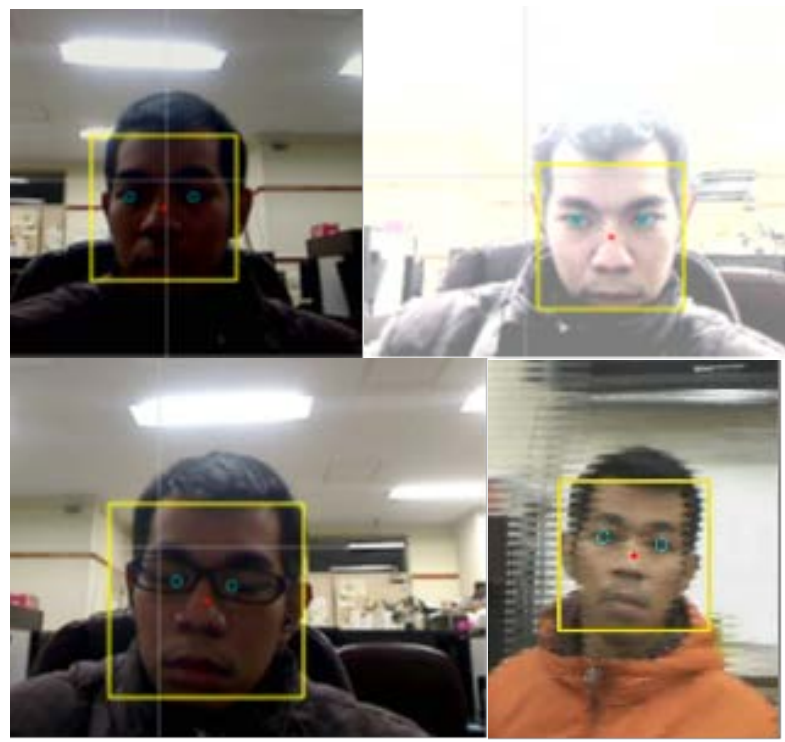

Fig.5. Example image of face and eye detection in different case; the rectangle is the face region and the circle is the position of the left and right eye.

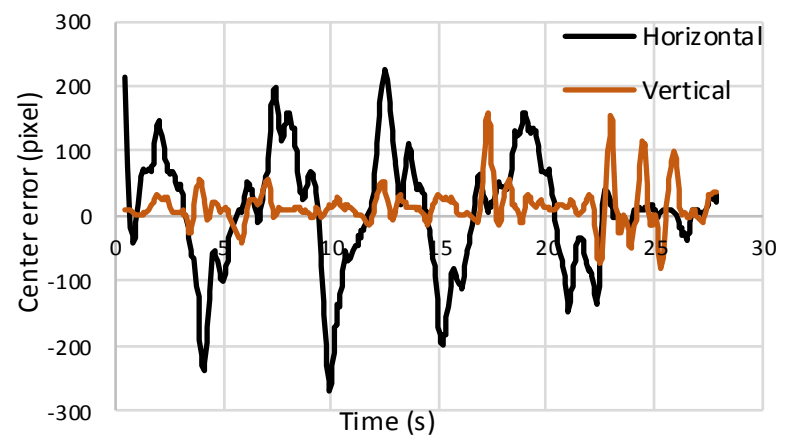

Fig.6. Distance error between the image center and tracking object center in horizontal and vertical

\section{Experiment}

In the system that we already provided, we experiment this system divided in two main sections; the first is face detection and recognition; and the second is the tracking system or the robot gaze following the recognized face. The face detection is implemented using local base pattern feature cascade classifier with the restricted condition of the smallest size image as $25 \times 25$ pixels which can detect the face. For the eye detection, we 
used Viola-Jones object detection framework with Haar feature-based cascade classifier that is an effective and popular object detection method [3]. We experiment the performance of the face and eye detection in different condition with some results as shown in Fig. 5. The face detection also is implemented to incorporate with the robot to experiment for the robot face tracking. The robot face tracking perform the experiment for the robot tracking performance as shown in Fig 6. In this experiment, we test the robot tracking the face while the human moving and facing the camera, the first we move from left to right side and return, the second we move up and down to test. The distance between the human while testing is around 1.5 meters. The experiment finds out the performance and the characteristic system such as the average frame rate as 16 frames per second, the average error as 5, 15.6 pixels, the minimum error as 272, -83 pixels and the maximum value as 228, 159 pixels in horizontal and vertical axis respectively.

The face recognition algorithm test with the laptop and the camera in the robot incorporating with the tracking control. In the experiment, 6 members of our lab have contributed the sample videos to construct the face recognition algorithm. The training program acquired the image by capturing each contributed videos. During the video recording using the camera in the head robot, the member would face the camera first then turn left, right and move up down his face in front of the camera slightly. Every image would perform the face detection using LBP-based cascade classifier profile and preprocessing image to preparing for training in Fisherfaces algorithm to construct the face recognition model [2], [5]. The training image size after resize and preprocessing would be $120 \times 120$ pixels.

Fig. 7 illustrates the example of the experimental results face recognition and tracking. There are two member lab testing the system, left side image which is the recognized face then the robot would track the face whereas the right side image which is the unknown face then the robot would not track the face. Fig 8 shows the results of the recognized face tracking such as the error of the center image between the center of the face region and the center of the camera image in horizontal axis and vertical axis. In this condition we move the face on the right, left, up and down to find out the characteristic and performance of the tracking control. In this experiment, we find out the average frame rate at 13 frames per second, a minimum error as $-121,-59$ pixels, a maximum error as 158, 84 pixels, and the average error as 2.7, 13.8 pixels in horizontal and vertical respectively.

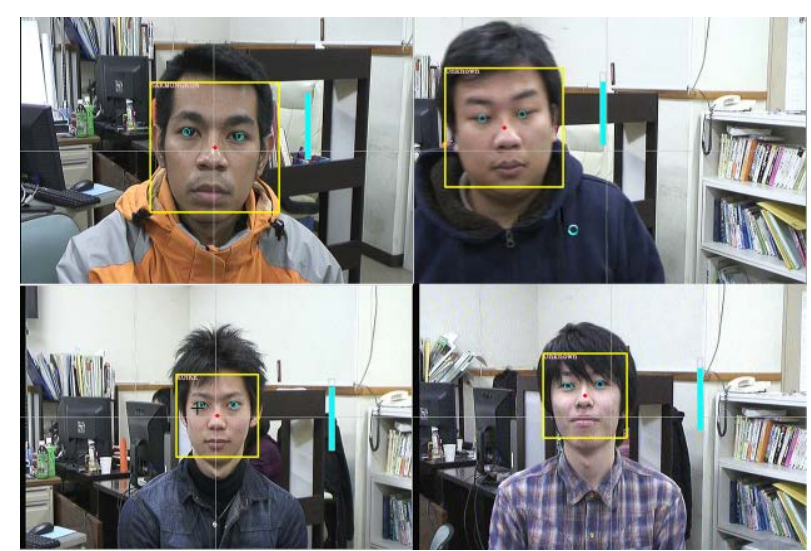

Fig.7. Example images in the robot tracking of recognized face. The left side show the recognized face tracking and the right side show unknown face.

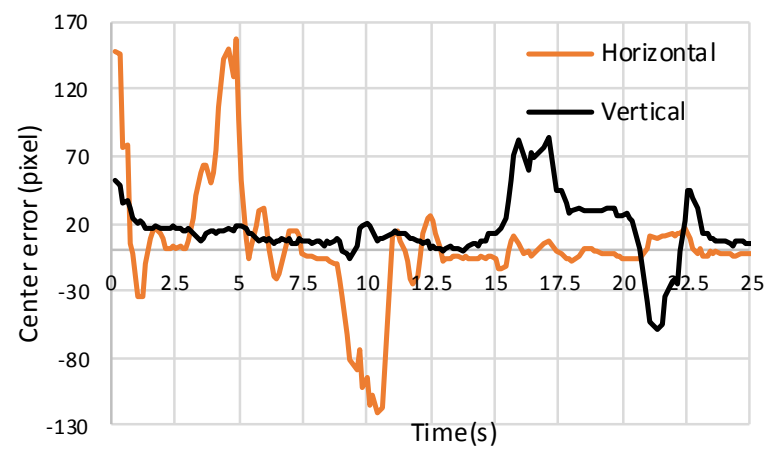

Fig.8. Distance error between the image center and tracking object center in horizontal and vertical.

\section{Conclusion}

This paper presents the face recognition combining with image tracking by CONBE robot for an experimental personal robot in our concept to develop the CBA. In the proposed visual tracking and the system processes around 16 frames per second for face detection and 13 frames per second for face recognition incorporating with robot face tracking. For the face detection and tracking the robot is able to track the face following pretty fast but in the recognized face tracking, the robot following pretty slow as the face recognition algorithm processing time and depending on the difficulty of the facial similarity. Because while testing the recognized face tracking, this system could not recognize the face 
every frame as the image is a blur image from the movement reason.

As the results, we first proceed the system to improve the robust face recognition in real time as well as possible. We then develop the facial express recognition to recognize the emotion expressing on the face to fulfill the part of the CBA operating with the face.

\section{Acknowledgements}

We would like to thank the members of our lab who contributed the image along with for testing the system and thank Mr. Masato Koike from Department of Mechanical Information Science and Technology, Kyushu Institute of technology who contributed the sample source code for the robot eye expression.

\section{References}

1. Hayashi E, Ueyama K, Yoshida M (2011), Autonomous motion selection via consciousness-based architecture. In Ubiquitous Robots and Ambient Intelligence (URAI), 2011 8th International Conference on, pp. 401-402

2. Belhumeur P. N., Hespanha J. P., Kriegman D. J. (1997), Eigenfaces vs. fisherfaces: Recognition using class specific linear projection. In Pattern Analysis and Machine Intelligence, IEEE Transactions on, pp. 711720.

3. Viola P., Jones M. (2001), Rapid object detection using a boosted cascade of simple features. In Computer Vision and Pattern Recognition, Proceedings of the 2001 IEEE Computer Society Conference on Vol. 1, pp. 511-518

4. Turk M, Pentland A (1991), Eigenfaces for recognition. In Journal of cognitive neuroscience, pp. 71-86.

5. He DC, Wang L. (1990), Texture Unit, Texture Spectrum, and Texture Analysis. In Geoscience and Remote Sensing, IEEE Transactions on, vol. 28, pp. 509 512. 